\title{
Congestion Games with Linearly Independent Paths: Convergence Time and Price of Anarchy ${ }^{\star}$
}

\author{
Dimitris Fotakis \\ Dept. of Information and Communication Systems Engineering \\ University of the Aegean, 83200 Samos, Greece. \\ fotakis@aegean.gr
}

\begin{abstract}
We investigate the effect of linear independence in the strategies of congestion games on the convergence time of best improvement sequences and on the pure Price of Anarchy. In particular, we consider symmetric congestion games on extension-parallel networks, an interesting class of networks with linearly independent paths, and establish two remarkable properties previously known only for parallel-link games. More precisely, we show that for arbitrary (non-negative and non-decreasing) latency functions, any best improvement sequence reaches a pure Nash equilibrium in at most as many steps as the number of players, and that for latency functions in class $\mathcal{D}$, the pure Price of Anarchy is at most $\rho(\mathcal{D})$. As a by-product of our analysis, we obtain that for symmetric congestion games on general networks with latency functions in class $\mathcal{D}$, the Price of Stability is at most $\rho(\mathcal{D})$.
\end{abstract}

Keywords: Network Congestion Games, Best Response Dynamics, Price of Anarchy, Price of Stability.

\section{Introduction}

Congestion games provide a natural model for non-cooperative resource allocation in large-scale communication networks and have been the subject of intensive research in algorithmic game theory. In a congestion game, a finite set of non-cooperative players, each controlling an unsplittable unit of load, compete over a finite set of resources. All players using a resource experience a latency (or cost) given by a non-negative and non-decreasing function of the resource's load (or congestion). Among a given set of resource subsets (or strategies), each player selects one selfishly trying to minimize her individual cost, that is the sum of the latencies on the resources in the chosen strategy. A natural solution concept is that of a pure Nash equilibrium, a configuration where no player can decrease her individual cost by unilaterally changing her strategy.

The prevailing questions in recent work on congestion games have to do with quantifying the inefficiency due to the players' selfish behaviour (see e.g. [22, 23, 17, 6, 9, 10, 4, 7]), and bounding the convergence time to pure Nash equilibria if the players select their strategies in a selfish and decentralized fashion (see e.g. [14, 21, 1, 8]). In this work, we investigate the effect of linear independence in the strategies of congestion games on the convergence time of best improvement sequences and on the inefficiency of pure Nash equilibria. In particular, we consider symmetric congestion games on extension-parallel networks, an interesting class of networks whose paths are linearly independent in the sense that every path contains an edge not included in any other path. For this class of congestion games, which comprises a natural and non-trivial generalization of the extensively studied class of

\footnotetext{
* A preliminary version of this work appeared in the Proceedings of the 1st International Symposium on Algorithmic Game Theory (SAGT 2008), B. Monien and U.-P. Schroeder (Eds.), Lecture Notes in Computer Science 4997, pp. 3345, Springer, 2008.
} 
parallel-link games (see e.g. [22, 23, 17, 14, 21,7]), we provide best possible answers to both research questions above.

Convergence Time to Pure Nash Equilibria. In a seminal paper, Rosenthal [26] proved that the pure Nash equilibria of congestion games correspond to the local optima of a natural potential function. Hence Rosenthal established that every congestion game admits at least one pure Nash equilibrium reached in a natural way when players iteratively make best improvement moves, i.e. adopt strategies that minimize their individual cost given the strategies of other players. Nevertheless, this may take an exponential number of steps, since computing a pure Nash equilibrium is PLS-complete even for nonsymmetric network congestion games as shown by Fabrikant et al. [15]. In fact, the proof of Fabrikant et al. establishes the existence of instances where any sequence of players' best improvement moves is exponentially long. Even for symmetric network congestion games, where a pure Nash equilibrium can be found efficiently by a min-cost flow computation [15], Ackermann et al. [1] presented a class of instances where any best improvement sequence is exponentially long.

A natural approach to circumvent the strong negative results of $[15,1]$ is to resort to approximate pure Nash equilibria, where no player can significantly improve her individual cost by unilaterally changing her strategy. Chien and Sinclair [8] considered symmetric congestion games with a weak restriction on latency functions and proved that several natural families of best improvement sequences converge to an approximate equilibrium in polynomial time.

An orthogonal approach is to identify interesting classes of congestion games for which best improvement sequences reach a pure Nash equilibrium in a polynomial number of steps. For instance, it is well known that for symmetric singleton congestion games (aka parallel-link games) with nonnegative and non-decreasing latency functions, any best improvement sequence reaches a pure Nash equilibrium in at most $n$ steps, where $n$ denotes the number of players. Ieong et al. [21] proved that even for non-symmetric singleton games with non-monotonic latencies, best improvement sequences reach a pure Nash equilibrium in a polynomial number of steps. Subsequently, Ackermann et al. [1] generalized this result to matroid congestion games, where the strategy space of each player consists of the bases of a matroid over the set of resources. Furthermore, Ackermann et al. proved that the matroid property on the players' strategy spaces is necessary for guaranteeing polynomial convergence of best improvement sequences if one does not take into account the global structure of the game.

Contribution. The negative results of $[15,1]$ leave open the possibility that some particular classes of symmetric network congestion games can guarantee fast convergence of best improvement sequences to pure Nash equilibria. In Section 3, we prove that for symmetric congestion games on extension-parallel networks with arbitrary (non-negative and non-decreasing) latency functions, any best improvement sequence reaches a pure Nash equilibrium in at most $n$ steps $^{1}$ (Theorem 1 ). In particular, we show that in any best improvement sequence, every player moves at most once (Lemma 1). This result is best possible, since there are instances where reaching a pure Nash equilibrium requires

\footnotetext{
${ }^{1}$ We highlight that matroid games and games on extension-parallel networks have a different combinatorial structure and may have quite different properties. For example, a network consisting of two parallel-link networks connected in series is not extension-parallel, while the corresponding network congestion game is a symmetric matroid game. For another example, Milchtaich [25, Example 4] proved that weighted congestion games on extension-parallel networks may not admit a pure Nash equilibrium. On the other hand, Ackermann et al. [2, Theorem 2] proved that every weighted matroid congestion game admits at least one pure Nash equilibrium.
} 
that every player moves at least once. To the best of our knowledge, symmetric congestion games on extension-parallel networks is the only class of congestion games other than parallel-link games for which such a strong bound on the convergence time of best improvement sequences is known.

Inefficiency of Pure Nash Equilibria. Having reached a pure Nash equilibrium, selfish players enjoy a minimum individual cost given the strategies of other players. However, the public benefit is usually measured by the total cost incurred by all players. Since a pure Nash equilibrium does not need to minimize the total cost, one seeks to quantify the inefficiency due to the players' non-cooperative and selfish behaviour. The Price of Anarchy, introduced by Koutsoupias and Papadimitriou [22], and the Price of Stability, introduced by Anshelevich et al. [5], are two widely accepted measures of the performance degradation due to the players' non-cooperative and selfish behaviour. The (pure) Price of Anarchy (PoA) (resp. Price of Stability, PoS) is the worst-case (resp. best-case) ratio of the total cost of a (pure) Nash equilibrium to the optimal total cost.

Many recent contributions have provided strong upper and lower bounds on the pure Price of Anarchy for several classes of congestion games, mostly congestion games with affine and polynomial latency functions and congestion games on parallel links ${ }^{2}$. Lücking et al. [23] were the first to consider the PoA of congestion games for the objective of total cost. For the special case of parallel links with linear latency functions, they proved that the $\mathrm{PoA}$ is $4 / 3$. For parallel links with polynomial latency functions of degree $d$, Gairing et al. [17] proved that the PoA is at most $d+1$. Awerbuch et al. [6] and Christodoulou and Koutsoupias [9] proved independently that the PoA of congestion games is $5 / 2$ for affine latency functions and $d^{\Theta(d)}$ for polynomial latency functions of degree $d$. Subsequently, Aland et al. [4] obtained exact bounds on the PoA of congestion games with polynomial latency functions. In the non-atomic setting, where the number of players is infinite and each player controls an infinitesimal amount of load, Roughgarden [27] proved that the PoA is independent of the strategy space and equal to $\rho(\mathcal{D})$, where $\rho$ depends on the class of latency functions $\mathcal{D}$ only (e.g. $\rho$ is equal to $4 / 3$ for affine and $\frac{27+6 \sqrt{3}}{23}$ for quadratic functions). Subsequently, Correa et al. [11] introduced $\beta(\mathcal{D})=1-\frac{1}{\rho(\mathcal{D})}$ and gave a simple proof of the same bound. Recently Fotakis [16] and independently Caragiannis et al. [7, Theorem 23] proved that the PoA of (atomic) congestion games on parallel links with latency functions in class $\mathcal{D}$ is $\rho(\mathcal{D})$, i.e. equal to the PoA of non-atomic congestion games with arbitrary strategies.

There is no difference between the Price of Anarchy and the Price of Stability for non-atomic congestion games, since the Nash equilibrium in the non-atomic setting is essentially unique (under mild assumptions on the latency functions, see e.g. [28]). In the atomic setting, Christodoulou and Koutsoupias [10] proved that the PoS of congestion games with affine latencies lies between $1+\sqrt{3} / 3$ and 1.6. Subsequently, Caragiannis et al. [7, Theorem 6] proved that the PoS of affine congestion games is $1+\sqrt{3} / 3$, and that for non-symmetric singleton games with latency functions in class $\mathcal{D}$, the $\operatorname{PoS}$ is at most $\rho(\mathcal{D})$.

Contribution. Despite the considerable interest in the PoA and the PoS of congestion games, it remains open whether better upper bounds close to $\rho$ are possible for symmetric games on simple networks other than parallel links (e.g. extension-parallel networks, series-parallel networks), or strong

\footnotetext{
${ }^{2}$ Here we cite only the most relevant results on the pure PoA for the objective of total cost. For a survey on the PoA of congestion games for the objective of total cost and the objective of maximum cost, see e.g. [18].
} 
lower bounds similar to the lower bounds of $[6,9,10,4]$ also apply to them. As a first step in this direction, we prove in Section 4 that the PoA of symmetric congestion games on extension-parallel networks with latency functions in class $\mathcal{D}$ is at most $\rho(\mathcal{D})$ (Theorem 3). On the negative side, we show that this result cannot be further generalized to series-parallel networks. In particular, we present an example of a 3-player symmetric game on a simple series-parallel network with linear latencies for which the $\mathrm{PoA}$ is $15 / 11$, greater than $4 / 3$, i.e. the value of $\rho$ for linear latencies. To the best of our knowledge, symmetric games on extension-parallel networks is the only class of congestion games other than parallel-link games for which such a strong bound on the pure Price of Anarchy is known.

To establish the upper bound of $\rho(\mathcal{D})$ on the PoA of symmetric games on extension-parallel networks, we show two properties of symmetric games that may be of independent interest. In particular, we prove that (i) for symmetric games on general networks with latencies in class $\mathcal{D}$, the total cost of any configuration minimizing Rosenthal's potential function is at most $\rho(\mathcal{D})$ times the optimal total cost (Lemma 2 and Lemma 3), and that (ii) for symmetric games on extension-parallel networks, every pure Nash equilibrium is a global minimum of Rosenthal's potential function (Lemma 4, also implicit in the work of Holzman and Law-Yone [19, Theorem 6.1]).

By the first property, we obtain an upper bound of $\rho(\mathcal{D})$ on the PoS of symmetric congestion games on general networks with latency functions in class $\mathcal{D}$ (Theorem 2). For instance, this implies that the PoS of symmetric network games with affine latencies is at most $4 / 3$, i.e. considerably better than the bound of $1+\sqrt{3} / 3$ known for general congestion games. To the best of our knowledge, the upper bound of $\rho(\mathcal{D})$ is the first bound on the Price of Stability that concerns a large natural class of congestion games other than singleton games and applies to arbitrary latency functions.

Related Work on Congestion Games with Linearly Independent Strategies. There has been a significant volume of previous work investigating the impact of linearly independent strategies on properties of congestion games. In [19], Holzman and Law-Yone proved that a strategy space admits a strong equilibrium ${ }^{3}$ for any selection of (non-negative and non-decreasing) latency functions if and only if it consists of linearly independent strategies. Furthermore, Holzman and Law-Yone showed that for symmetric congestion games with linearly independent strategies, every pure Nash equilibrium is a strong equilibrium. Subsequently, Holzman and Law-Yone [20] proved that the class of congestion games on extension-parallel networks is the network equivalent of congestion games with linearly independent strategies.

Milchtaich [24] was the first to consider networks with linearly independent paths (under this name). Milchtaich proved that an undirected network has linearly independent paths if and only if it is extension-parallel. Moreover, Milchtaich showed that networks with linearly independent paths is the only class of networks where for any selection of non-negative and increasing (resp. non-decreasing) latency functions, all equilibria in the non-atomic setting are Pareto (resp. weakly Pareto) efficient.

Recently Epstein et al. [13,12] considered fair connection games and congestion games on extension-parallel networks. In [13], Epstein et al. proved that fair connection games on extensionparallel networks admit a strong equilibrium. In [12], Epstein et al. showed that extension-parallel networks is the only class of networks where for any selection of (non-negative and non-decreasing) latency functions, every pure Nash equilibrium minimizes the maximum players' cost.

\footnotetext{
${ }^{3}$ The notion of strong equilibrium was introduced by Aumann in late 50's. A configuration is a strong equilibrium if no coalition of players can deviate in a way profitable for all its members.
} 


\section{Model and Preliminaries}

For any integer $k \geq 1$, we denote $[k] \equiv\{1, \ldots, k\}$. For a vector $x=\left(x_{1}, \ldots, x_{n}\right)$, we denote $x_{-i} \equiv\left(x_{1}, \ldots, x_{i-1}, x_{i+1}, \ldots, x_{n}\right)$ and $\left(x_{-i}, x_{i}^{\prime}\right) \equiv\left(x_{1}, \ldots, x_{i-1}, x_{i}^{\prime}, x_{i+1}, \ldots, x_{n}\right)$.

Congestion Games. A congestion game is a tuple $\Gamma\left(N, E,\left(\Sigma_{i}\right)_{i \in N},\left(d_{e}\right)_{e \in E}\right)$, where $N$ denotes the set of players, $E$ denotes the set of resources, $\Sigma_{i} \subseteq 2^{E} \backslash\{\emptyset\}$ denotes the strategy space of each player $i$, and $d_{e}: \mathbb{N} \mapsto \mathbb{R}_{\geq 0}$ is a non-negative and non-decreasing latency function associated with each resource $e$. A congestion game is symmetric if all players have a common strategy space. In the following, we let $n$ denote the number of players.

A configuration is a vector $\sigma=\left(\sigma_{1}, \ldots, \sigma_{n}\right)$ consisting of a strategy $\sigma_{i} \in \Sigma_{i}$ for each player $i$. For every resource $e$, we let $\sigma_{e}=\left|\left\{i \in N: e \in \sigma_{i}\right\}\right|$ denote the congestion induced on $e$ by $\sigma$. The individual cost of player $i$ in the configuration $\sigma$ is $c_{i}(\sigma)=\sum_{e \in \sigma_{i}} d_{e}\left(\sigma_{e}\right)$. A configuration $\sigma$ is a pure Nash equilibrium if no player can improve her individual cost by unilaterally changing her strategy. Formally, $\sigma$ is a Nash equilibrium if for every player $i$ and every strategy $s_{i} \in \Sigma_{i}, c_{i}(\sigma) \leq c_{i}\left(\sigma_{-i}, s_{i}\right)$.

Rosenthal [26] introduced the potential function

$$
\Phi(\sigma)=\sum_{e \in E} \sum_{i=1}^{\sigma_{e}} d_{e}(i),
$$

and proved that when a player $i$ switches from her current strategy $\sigma_{i}$ to a new strategy $s_{i} \in \Sigma_{i}$, the difference in the potential value equals the difference in the individual cost of player $i$, i.e.

$$
\Phi\left(\sigma_{-i}, s_{i}\right)-\Phi(\sigma)=c_{i}\left(\sigma_{-i}, s_{i}\right)-c_{i}(\sigma)
$$

Thus Rosenthal established that congestion games (even with non-monotonic latency functions) admit a pure Nash equilibrium, and that the pure Nash equilibria of a congestion game correspond to the local minima of the potential function $\Phi$. For symmetric network congestion games with arbitrary (non-negative and non-decreasing) latency functions, Fabrikant et al. [15, Theorem 2] proved that the global minimum of the potential function $\Phi$, and thus a pure Nash equilibrium, can be computed in polynomial time by a min-cost flow computation.

In this work, we focus on symmetric network congestion games, where the players' strategies are determined by a directed network $G(V, E)$ with a distinguished source $s$ and sink $t$ (aka a $s-t$ network). The network edges play the role of resources and the common strategy space of the players is the set of (simple) $s-t$ paths in $G$, denoted $\mathcal{P}$. For any $s-t$ path $p$ and any pair of vertices $v_{1}, v_{2}$ on $p$, we let $p\left[v_{1}, v_{2}\right]$ denote the segment of $p$ between vertices $v_{1}$ and $v_{2}\left(p\left[v_{1}, v_{2}\right]\right.$ is empty if $v_{1}$ appears after $v_{2}$ on $p$ ). For consistency with the definition of strategies as resource subsets, we usually regard paths as sets of edges.

Flows and Configurations. Let $G(V, E)$ be a directed network with source $s$ and sink $t$. A $s-t$ flow $f$ is a vector $\left(f_{e}\right)_{e \in E} \in \mathbb{R}_{\geq 0}^{m}$ that satisfies the flow conservation at all vertices other than $s$ and $t$. The volume of $f$ is the total flow leaving $s$. A flow $f$ is acyclic if there is no directed cycle in $G$ with positive flow on all its edges. For a flow $f$ and a path $p \in \mathcal{P}$, we let $f_{p}^{\min } \equiv \min _{e \in p}\left\{f_{e}\right\}$.

Given a configuration $\sigma$ of a symmetric network congestion game $\Gamma$, we refer to the congestion vector $\left(\sigma_{e}\right)_{e \in E}$ as the $s-t$ flow induced by $\sigma$. We say that a flow $f$ is feasible if there is a configuration 
inducing congestion $f_{e}$ on every edge $e$. Hence any configuration of $\Gamma$ corresponds to a feasible flow, while a feasible flow may be induced by many different configurations. Moreover, every integer acyclic $s-t$ flow of volume $n$ corresponds to (possibly many) configurations of $\Gamma$, and thus is feasible. We say that a configuration is acyclic if the corresponding feasible flow is acyclic. In the following, we slightly abuse the notation by letting the same symbol denote both a configuration and the feasible flow induced by it.

Best Improvement Sequences. A strategy $s_{i} \in \Sigma_{i}$ is a best response of player $i$ to a configuration $\sigma$ (or equivalently to $\sigma_{-i}$ ) if for every strategy $s_{i}^{\prime} \in \Sigma_{i}, c_{i}\left(\sigma_{-i}, s_{i}\right) \leq c_{i}\left(\sigma_{-i}, s_{i}^{\prime}\right)$. If the current strategy $\sigma_{i}$ of player $i$ is not a best response to the current configuration $\sigma$, a best response of $i$ to $\sigma$ is called a best improvement of player $i$. We consider best improvement sequences, where in each step, a player $i$ whose strategy $\sigma_{i}$ is not a best response to the current configuration $\sigma$ switches to her best improvement. The existence of the potential function $\Phi$ implies that any best improvement sequence converges to a pure Nash equilibrium in a finite number of steps. In the first part of this work, we are interested in bounding the maximum length of best improvement sequences for symmetric congestion games on extension-parallel networks.

Social Cost, Price of Anarchy, and Price of Stability. In the second part of this work, we are interested in quantifying the inefficiency of pure Nash equilibria for symmetric congestion games on extension-parallel networks and on general networks. We evaluate configurations using the objective of total cost. The total cost $C(\sigma)$ of a configuration $\sigma$ is the sum of players' individual costs in $\sigma$, i.e.

$$
C(\sigma)=\sum_{i=1}^{n} c_{i}(\sigma)=\sum_{e \in E} \sigma_{e} d_{e}\left(\sigma_{e}\right)
$$

The optimal configuration, denoted $o$, minimizes the total cost among all configurations in $\mathcal{P}^{n}$.

The pure Price of Anarchy (PoA) of a congestion game $\Gamma$ is the maximum ratio $C(\sigma) / C(o)$ over all pure Nash equilibria $\sigma$ of $\Gamma$. The pure Price of Stability (PoS) of $\Gamma$ is the minimum ratio $C(\sigma) / C(o)$ over all pure Nash equilibria $\sigma$ of $\Gamma$. In other words, the pure PoA (resp. PoS) is equal to $C(\sigma) / C(o)$, where $\sigma$ is $\Gamma$ 's pure Nash equilibrium of maximum (resp. minimum) total cost. Hence the pure PoA (resp. PoS) of $\Gamma$ is bounded from above by a number $\alpha$ if all pure Nash equilibria (resp. some pure Nash equilibrium) of $\Gamma$ have (resp. has) total cost at most $\alpha$ times the optimal total cost.

We use the quantities $\rho(\mathcal{D})$ and $\beta(\mathcal{D})$ introduced in [27, 11] respectively, to bound the PoS of symmetric network congestion games and the PoA of symmetric games on extension-parallel networks. For a non-negative and non-decreasing function $d(x)$, let $\rho(d) \equiv \sup _{x \geq y \geq 0} \frac{x d(x)}{y d(y)+(x-y) d(x)}$, and let $\beta(d) \equiv \sup _{x \geq y \geq 0} \frac{y(d(x)-d(y))}{x d(x)}$. For a non-empty class of non-negative and non-decreasing functions $\mathcal{D}$, let $\rho(\mathcal{D}) \equiv \sup _{d \in \mathcal{D}} \rho(d)$, and let $\beta(\mathcal{D}) \equiv \sup _{d \in \mathcal{D}} \beta(d)$. We note that $(1-\beta(\mathcal{D}))^{-1}=\rho(\mathcal{D})$.

Extension-Parallel Networks. Let $G_{1}\left(V_{1}, E_{1}\right)$ and $G_{2}\left(V_{2}, E_{2}\right)$ be two networks with source $s_{1} \in V_{1}$ and $s_{2} \in V_{2}$ and sink $t_{1} \in V_{1}$ and $t_{2} \in V_{2}$ respectively, and let $G^{\prime}\left(V_{1} \cup V_{2}, E_{1} \cup E_{2}\right)$ be the union network of $G_{1}$ and $G_{2}$. The parallel composition of $G_{1}$ and $G_{2}$ results in a $s-t$ network obtained from $G^{\prime}$ by identifying $s_{1}$ and $s_{2}$ to the source $s$ and identifying $t_{1}$ and $t_{2}$ to the sink $t$. The series composition of $G_{1}$ and $G_{2}$ results in a $s-t$ network obtained from $G^{\prime}$ by letting $s_{1}$ be the source $s$, letting $t_{2}$ be the sink $t$, and identifying $t_{1}$ with $s_{2}$. 
A directed $s-t$ network is series-parallel if it consists of either a single edge $(s, t)$ or two seriesparallel networks composed either in series or in parallel. A directed $s-t$ network is extension-parallel if it consists of either: (i) a single edge $(s, t)$, (ii) a single edge and an extension-parallel network composed in series, or (iii) two extension-parallel networks composed in parallel. Every extensionparallel network is series-parallel. The converse is true only if in every series composition, at least one component is a single edge.

The following proposition gives a useful property of series-parallel (and thus of extension-parallel) networks. For completeness, we prove the proposition in the Appendix, Section A.1.

Proposition 1. Let $G(V, E)$ be a series-parallel $s-t$ network, and let $u, w$ be two vertices connected by two internally disjoint paths $p$ and $p^{\prime}$. Then every $s-t$ path having an edge in common with $p$ contains both $u$ and $w$ and does not intersect $p^{\prime}$ at any vertex other than $u$ and $w$.

A $s-t$ network has linearly independent paths if every $s-t$ path contains at least one edge not belonging to any other $s-t$ path $^{4}$. Milchtaich [24, Proposition 5] proved that an undirected $s-t$ network has linearly independent paths if and only if it is extension-parallel. Therefore, every (directed) extension-parallel network has linearly independent paths (see also [20, Theorem 1]). Furthermore, [24, Propositions 3 and 5] imply that a (directed) series-parallel network has linearly independent paths if and only if it is extension-parallel.

The following proposition gives a useful property of extension-parallel networks (for a proof, see [24, Proposition 4]).

Proposition 2 (Milchtaich). Let $G$ be an extension-parallel $s-t$ network. Then for every pair of $s-t$ paths $p$ and $p^{\prime}$ in $G$, and every vertex $v$ common to both paths, $p$ and $p^{\prime}$ share either the segment between $s$ and $v$ or the segment between $v$ and $t$.

Proposition 2 implies that for any two paths $p$ and $p^{\prime}$ of an extension-parallel network, the segments $p \backslash p^{\prime}$ and $p^{\prime} \backslash p$ where $p$ and $p^{\prime}$ deviate from each other are two internally disjoint paths with common endpoints.

The following proposition gives an interesting property of networks with linearly independent paths (and thus of extension-parallel networks).

Proposition 3. Let $\Gamma$ be a symmetric congestion game on a s-t network $G$ with linearly independent paths, let $f$ be any configuration of $\Gamma$, and let $\pi$ be any (simple) path of $G$ with $f_{\pi}^{\min }>0$. Then there exists a player $i$ whose strategy in $f$ includes $\pi$.

Proof. We first consider the case where $\pi$ is a $s-t$ path. Since $G$ is a $s-t$ network with linearly independent paths, there exists an edge $e \in \pi$ not belonging to any other $s-t$ path. Let $i$ be any player whose strategy in $f$ includes $e$ (such a player exists because $f_{e}>0$ ). Then the strategy of player $i$ in $f$ is $\pi$ (since $e$ would not be included in $f_{i}$ otherwise).

If $\pi$ is not a $s-t$ path, let $u$ and $w$ be the endpoints of $\pi$. Since $f$ corresponds to a $s-t$ flow with $f_{\pi}^{\min }>0$, there is a $s-u$ path $\pi_{1}$ with $f_{\pi_{1}}^{\min }>0\left(\pi_{1}\right.$ is empty if $u$ is $\left.s\right)$ and a $w-t$ path $\pi_{2}$

\footnotetext{
${ }^{4}$ The name is motivated by the fact that in such a network, it is not possible to express any path as the symmetric difference of some other paths [24, Proposition 6].
} 
with $f_{\pi_{2}}^{\min }>0\left(\pi_{2}\right.$ is empty if $w$ is $\left.t\right)$. Then $p=\left(\pi_{1}, \pi, \pi_{2}\right)$ is a $s-t$ path with $f_{p}^{\text {min }}>0$. As shown before, there is a player $i$ whose strategy in $f$ is $p$, which includes $\pi$.

Every configuration of a symmetric congestion game on a series-parallel (and thus on an extension-parallel) network corresponds to a (feasible) acyclic flow of volume $n$. Proposition 3 implies that for every symmetric congestion game $\Gamma$ on an extension-parallel $s-t$ network, any acyclic $s-t$ flow of volume $n$ corresponds to a unique configuration of $\Gamma$ (uniqueness is up to players' permutation). Therefore, for symmetric congestion games on extension-parallel networks, there is a correspondence between configurations and feasible acyclic flows.

\section{Convergence Time to Pure Nash Equilibria}

In this section, we prove that for symmetric congestion games on extension-parallel networks, any best improvement sequence reaches a pure Nash equilibrium in at most $n$ steps. The proof is based on the following lemma establishing that in any best improvement sequence, every player moves at most once.

Lemma 1. Let $\Gamma$ be a symmetric congestion game on an extension-parallel network, let $\sigma$ be the current configuration, and let $i$ be a player switching from her current strategy $\sigma_{i}$ to her best improvement $\sigma_{i}^{\prime}$. Then for every player $j$ whose current strategy $\sigma_{j}$ is a best response to $\sigma, \sigma_{j}$ remains a best response of $j$ to the new configuration $\sigma^{\prime}=\left(\sigma_{-i}, \sigma_{i}^{\prime}\right)$.

Proof. For sake of contradiction, we assume that there is a player $j$ whose current strategy $\sigma_{j}$ is a best response to $\sigma$ but not to $\sigma^{\prime}$. Let $\sigma_{j}^{\prime}$ be the best response of $j$ to $\sigma^{\prime}$, and let $p=\sigma_{j} \backslash \sigma_{j}^{\prime}$ and $p^{\prime}=\sigma_{j}^{\prime} \backslash \sigma_{j}$ be the segments where $\sigma_{j}$ and $\sigma_{j}^{\prime}$ deviate from each other. Due to the extension-parallel structure of the network (see also Proposition 2), $p$ and $p^{\prime}$ are internally disjoint paths with common endpoints, denoted $u$ and $w$ ( $u$ may be $s$ and $w$ may be $t$ ).

Since $p$ and $p^{\prime}$ are edge-disjoint paths and player $j$ improves her individual cost in $\sigma^{\prime}$ by switching from $p$ to $p^{\prime}$,

$$
\sum_{e \in p} d_{e}\left(\sigma_{e}^{\prime}\right)>\sum_{e \in p^{\prime}} d_{e}\left(\sigma_{e}^{\prime}+1\right)
$$

Using the inequality above and the hypothesis that $\sigma_{i}^{\prime}$ is a best improvement of player $i$ to $\sigma$, and exploiting the extension-parallel structure of the network, we establish that if player $j$ prefers $\sigma_{j}^{\prime}$ to $\sigma_{j}$ in the new configuration $\sigma^{\prime}$, then $\sigma_{j}$ is not a best response of $j$ to $\sigma$. In particular, we show that player $j$ can also improve her individual cost in $\sigma$ by switching from an appropriate segment of $\sigma_{j}$ to the corresponding segment of $\sigma_{i}^{\prime}$. Clearly, this contradicts the hypothesis that $\sigma_{j}$ is a best response of $j$ to $\sigma$ and implies the lemma.

The technical part of the proof proceeds by case analysis. We first distinguish between two cases depending on whether $\sigma_{i}^{\prime}$ contains both $u$ and $w$ or not.

Case I, $\boldsymbol{u}, \boldsymbol{w} \in \boldsymbol{\sigma}_{\boldsymbol{i}}^{\prime}$ : We first consider the case where $\sigma_{i}^{\prime}$ contains $u$ and $w$ and thus $\sigma_{i}^{\prime}[u, w]$ can serve as an alternative to $p$. We further distinguish between two subcases depending on whether $p$ and $\sigma_{i}^{\prime}$ are edge-disjoint or not. 
Case I.a, $p \cap \sigma_{i}^{\prime}=\emptyset$ : We start with the case where $p$ and $\sigma_{i}^{\prime}$ are edge-disjoint. We first consider the case where $\sigma_{i}^{\prime}[u, w] \backslash p^{\prime}$ does not contain any edges of $\sigma_{i}$ (see e.g. Fig. 1.a). Then,

$$
\begin{aligned}
\sum_{e \in p^{\prime}} d_{e}\left(\sigma_{e}^{\prime}+1\right) & \geq \sum_{e \in p^{\prime} \cap \sigma_{i}^{\prime}} d_{e}\left(\sigma_{e}+1\right)+\overbrace{\sum_{e \in\left(p^{\prime} \cap \sigma_{i}\right) \backslash \sigma_{i}^{\prime}} d_{e}\left(\sigma_{e}\right)+\sum_{e \in\left(p^{\prime} \backslash \sigma_{i}\right) \backslash \sigma_{i}^{\prime}} d_{e}\left(\sigma_{e}+1\right)}^{\text {cost of player } i \text { on } p^{\prime} \backslash \sigma_{i}^{\prime} \text { in } \sigma} \\
& \geq \sum_{e \in p^{\prime} \cap \sigma_{i}^{\prime}} d_{e}\left(\sigma_{e}+1\right)+\underbrace{\sum_{e \in \sigma_{i}^{\prime}[u, w] \backslash p^{\prime}} d_{e}\left(\sigma_{e}+1\right)}_{\sum_{\text {cost of player } i \text { on } \sigma_{i}^{\prime}[u, w] \backslash p^{\prime} \text { in } \sigma} d_{e}\left(\sigma_{e}+1\right)} \\
& =\sum_{i, w]}
\end{aligned}
$$

For the first inequality, we use that when player $i$ switches from $\sigma_{i}$ to $\sigma_{i}^{\prime}$ : (i) the congestion of any edge $e$ in $\sigma_{i}^{\prime}$ does not decrease (i.e. $\forall e \in \sigma_{i}^{\prime}, \sigma_{e}^{\prime} \geq \sigma_{e}$ ), (ii) the congestion of any edge $e$ decreases by at most 1 (i.e. $\forall e, \sigma_{e}^{\prime} \geq \sigma_{e}-1$ ), and (iii) the congestion of any edge $e$ not in $\sigma_{i}$ or $\sigma_{i}^{\prime}$ does not change (i.e. $\forall e \notin \sigma_{i} \cup \sigma_{i}^{\prime}, \sigma_{e}^{\prime}=\sigma_{e}$ ). For the second inequality, we observe that the marked terms on the left-hand side is the individual cost of player $i$ on $p^{\prime} \backslash \sigma_{i}^{\prime}$ in $\sigma$ (i.e. when the configuration of the remaining players is $\sigma_{-i}$ ) and that the marked term on the right-hand side is the individual cost of player $i$ on $\sigma_{i}^{\prime}[u, w] \backslash p^{\prime}$ in $\sigma$ (recall that we consider the case where $\sigma_{i}^{\prime}[u, w] \backslash p^{\prime}$ does not contain any edges of $\sigma_{i}$ ). Since $\sigma_{i}^{\prime}$ is a best response of player $i$ to $\sigma_{-i}$, the former cost is no less than the latter.

Using (2), we conclude that player $j$ can improve her individual cost in $\sigma$ by changing her path between $u$ and $w$ from $p$ to $\sigma_{i}^{\prime}[u, w]$, which contradicts the hypothesis that $\sigma_{j}$ is a best response of player $j$ to $\sigma$. Formally,

$$
\sum_{e \in p} d_{e}\left(\sigma_{e}\right) \geq \sum_{e \in p} d_{e}\left(\sigma_{e}^{\prime}\right)>\sum_{e \in p^{\prime}} d_{e}\left(\sigma_{e}^{\prime}+1\right) \geq \sum_{e \in \sigma_{i}^{\prime}[u, w]} d_{e}\left(\sigma_{e}+1\right)
$$

The first inequality holds because $p \cap \sigma_{i}^{\prime}=\emptyset$. Therefore, for all $e \in p, \sigma_{e} \geq \sigma_{e}^{\prime}$, since the congestion of any edge not in $\sigma_{i}^{\prime}$ does not increase when player $i$ switches from $\sigma_{i}$ to $\sigma_{i}^{\prime}$. The second inequality is (1), and the third inequality is (2).

If $\sigma_{i}^{\prime}[u, w] \backslash p^{\prime}$ contains some edges of $\sigma_{i}$, we show that due to the extension-parallel structure of the network, the congestion of the edges in $p$ and $p^{\prime}$ does not change when player $i$ switches from $\sigma_{i}$ to $\sigma_{i}^{\prime}$ (see Fig. 1.b). This contradicts the hypothesis that the best response of player $j$ changes from $\sigma_{j}$ to $\sigma_{j}^{\prime}$ when player $i$ moves from $\sigma_{i}$ to $\sigma_{i}^{\prime}$.

We first show that the congestion of the edges of $p$ does not change when player $i$ switches from $\sigma_{i}$ to $\sigma_{i}^{\prime}$. Since $u$ and $w$ are connected by two internally disjoint paths $p$ and $\sigma_{i}^{\prime}[u, w]$, and since $\sigma_{i}$ and $\sigma_{i}^{\prime}[u, w]$ have some edges in common, by Proposition $1, \sigma_{i}$ contains $u$ and $w$ and does not have any edges in common with $p$. Hence the congestion of the edges of $p$ is the same in both $\sigma$ and $\sigma^{\prime}$.

Next we show that the congestion of the edges of $p^{\prime}$ does not change when player $i$ switches from $\sigma_{i}$ to $\sigma_{i}^{\prime}$. Let $\pi=\sigma_{i}^{\prime}[u, w] \backslash p^{\prime}$ and $\pi^{\prime}=p^{\prime} \backslash \sigma_{i}^{\prime}[u, w]$ be the segments where $\sigma_{i}^{\prime}[u, w]$ and $p^{\prime}$ deviate from each other. Since $\pi \cap \sigma_{i} \neq \emptyset, \pi$ and $\pi^{\prime}$ are non-empty. Thus they are internally disjoint paths with common endpoints, denoted $u^{\prime}$ and $w^{\prime}$. Their first endpoint $u^{\prime}$ appears no sooner than $u$ and their last endpoint $w^{\prime}$ appears no later than $w$ on $\sigma_{i}^{\prime}$ and $p^{\prime}$. Since $\sigma_{i}$ has some edges in common with $\pi$, 
(a)

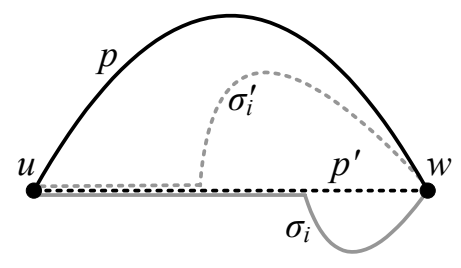

(d)

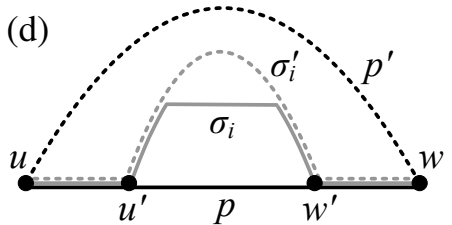

(b)

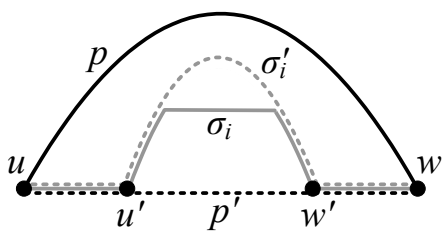

(c)

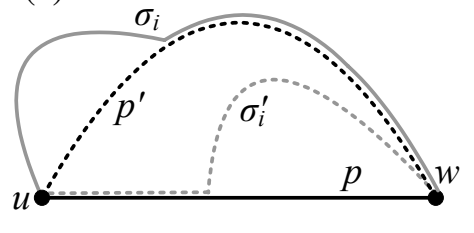

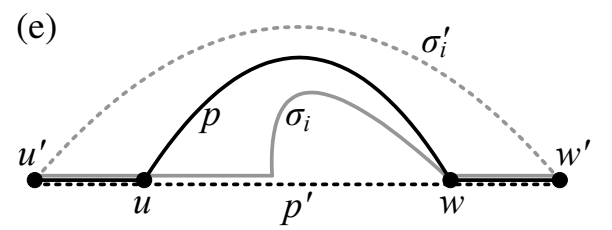

Fig. 1. The different cases considered in the proof of Lemma 1. In each case, the solid black path labeled $p$ represents the best response of player $j$ in $\sigma$ between vertices $u$ and $w$, the solid grey path labeled $\sigma_{i}$ represents the strategy of player $i$ in $\sigma$, and the dotted grey path labeled $\sigma_{i}^{\prime}$ represents the best response of player $i$ to $\sigma$. We assume that the best response of player $j$ changes from $p$ to the dotted black path labeled $p^{\prime}$ when player $i$ switches from $\sigma_{i}$ to $\sigma_{i}^{\prime}$, and establish a contradiction in all cases.

by Proposition 1, $\sigma_{i}$ contains $u^{\prime}$ and $w^{\prime}$ and does not intersect $\pi^{\prime}$ at any vertex other than $u^{\prime}$ and $w^{\prime}$. Moreover, since all three paths $p^{\prime}, \sigma_{i}^{\prime}[u, w]$ and $\sigma_{i}[u, w]$ contain $u^{\prime}$ and $w^{\prime}$, and since $\sigma_{i}^{\prime}[u, w]$ and $\sigma_{i}[u, w]$ deviate from $p^{\prime}$ between $u^{\prime}$ and $w^{\prime}$, by Proposition 2, the three paths share their $u-u^{\prime}$ and $w^{\prime}-w$ segments (see also Fig. 1.b). Therefore, if $\sigma_{i}^{\prime}[u, w]$ and $\sigma_{i}[u, w]$ deviate from each other, this happens between $u^{\prime}$ and $w^{\prime}$. Consequently, the corresponding path segments $\sigma_{i}^{\prime}\left[u^{\prime}, w^{\prime}\right]$ and $\sigma_{i}\left[u^{\prime}, w^{\prime}\right]$ do not contain any edges of $p^{\prime}$. Thus the congestion of the edges of $p^{\prime}$ does not change when player $i$ switches from $\sigma_{i}$ to $\sigma_{i}^{\prime}$.

Case I.b, $p \cap \sigma_{i}^{\prime} \neq \emptyset$ : We proceed with the case where $p$ and $\sigma_{i}^{\prime}$ are not edge-disjoint. Then, by Proposition $1, \sigma_{i}^{\prime}$ does not have any edges in common with $p^{\prime}$ and does not intersect $p^{\prime}$ at any vertex other than $u$ and $w$. We first consider the case where $\sigma_{i}^{\prime}[u, w] \backslash p$ does not contain any edges of $\sigma_{i}$ (see e.g. Fig. 1.c). Then,

$$
\begin{aligned}
\sum_{e \in p \cap \sigma_{i}^{\prime}} d_{e}\left(\sigma_{e}^{\prime}\right)+\sum_{e \in p \backslash \sigma_{i}^{\prime}} d_{e}\left(\sigma_{e}\right) \geq \sum_{e \in p} d_{e}\left(\sigma_{e}^{\prime}\right) & >\sum_{e \in p^{\prime}} d_{e}\left(\sigma_{e}^{\prime}+1\right) \\
& \geq \sum_{e \in p^{\prime} \cap \sigma_{i}} d_{e}\left(\sigma_{e}\right)+\sum_{e \in p^{\prime} \backslash \sigma_{i}} d_{e}\left(\sigma_{e}+1\right) \\
& \geq \sum_{e \in \sigma_{i}^{\prime}[u, w]} d_{e}\left(\sigma_{e}^{\prime}\right) \\
& =\sum_{e \in p \cap \sigma_{i}^{\prime}} d_{e}\left(\sigma_{e}^{\prime}\right)+\sum_{e \in \sigma_{i}^{\prime}[u, w] \backslash p} d_{e}\left(\sigma_{e}+1\right)
\end{aligned}
$$

The first inequality holds because the congestion of any edge not in $\sigma_{i}^{\prime}$ does not increase when player $i$ switches from $\sigma_{i}$ to $\sigma_{i}^{\prime}$ (i.e. $\forall e \notin \sigma_{i}^{\prime}, \sigma_{e} \geq \sigma_{e}^{\prime}$ ). The second inequality is (1). The third inequality holds because when player $i$ switches from $\sigma_{i}$ to $\sigma_{i}^{\prime}$ : (i) the congestion of any edge decreases by at most 1 (i.e. $\forall e, \sigma_{e}^{\prime} \geq \sigma_{e}-1$ ), and (ii) the congestion of any edge not in $\sigma_{i}$ does not decrease (i.e. $\forall e \notin \sigma_{i}$, 
$\sigma_{e}^{\prime} \geq \sigma_{e}$ ). For the fourth inequality, we observe that the left-hand side is equal to the individual cost of player $i$ on $p^{\prime}$ in $\sigma$ (i.e. when the configuration of the remaining players is $\sigma_{-i}$ ), and that the right-hand side is equal to the cost of player $i$ on $\sigma_{i}^{\prime}[u, w]$ in $\sigma$. Since $\sigma_{i}^{\prime}$ is a best response of player $i$ to $\sigma_{-i}$, the former cost is not less than the latter. The equality holds because $\sigma_{i}^{\prime}[u, w] \backslash p$ does not contain any edges of $\sigma_{i}$ and thus the congestion of every edge in $\sigma_{i}^{\prime}[u, w] \backslash p$ increases by 1 when player $i$ switches from $\sigma_{i}$ to $\sigma_{i}^{\prime}$ (i.e. $\forall e \in \sigma_{i}^{\prime}[u, w] \backslash p, \sigma_{e}^{\prime}=\sigma_{e}+1$ ). Thus (3) implies that

$$
\sum_{e \in p \backslash \sigma_{i}^{\prime}} d_{e}\left(\sigma_{e}\right)>\sum_{e \in \sigma_{i}^{\prime}[u, w] \backslash p} d_{e}\left(\sigma_{e}+1\right)
$$

Therefore, player $j$ can improve her individual cost in $\sigma$ by switching from $p \backslash \sigma_{i}^{\prime}$ to $\sigma_{i}^{\prime}[u, w] \backslash p$. This contradicts the hypothesis that $\sigma_{j}$ is a best response of player $j$ to $\sigma$.

The case where $\sigma_{i}^{\prime}[u, w] \backslash p$ contains some edges of $\sigma_{i}$ (see e.g. Fig. 1.d) is symmetric to the subcase of I.a where $\sigma_{i}^{\prime}[u, w] \backslash p^{\prime}$ contains some edge of $\sigma_{i}$. Applying the same arguments to the paths $\sigma_{i}^{\prime}[u, w], \sigma_{i}[u, w]$ and $p$ (instead of $p^{\prime}$ in the subcase of I.a), we show that the congestion of the edges in $p$ and $p^{\prime}$ does not change when player $i$ switches from $\sigma_{i}$ to $\sigma_{i}^{\prime}$. This contradicts the hypothesis that the best response of player $j$ changes from $\sigma_{j}$ to $\sigma_{j}^{\prime}$ when player $i$ moves from $\sigma_{i}$ to $\sigma_{i}^{\prime}$. For completeness, we include the details in the Appendix, Section A.2.

Case II, either $\boldsymbol{u} \notin \boldsymbol{\sigma}_{\boldsymbol{i}}^{\prime}$ or $\boldsymbol{w} \notin \boldsymbol{\sigma}_{\boldsymbol{i}}^{\prime}$ : We proceed with the case where $\sigma_{i}^{\prime}$ does not contain at least one of $u$ and $w$. Then, by Proposition $1, \sigma_{i}^{\prime}$ does have any edges in common with both $p$ and $p^{\prime}$.

If $\sigma_{i}$ too does not contain at least one of $u$ and $w$, then $\sigma_{i}$ does not have any edges in common with both $p$ and $p^{\prime}$. Therefore, the congestion of the edges in $p$ and $p^{\prime}$ does not change when player $i$ switches from $\sigma_{i}$ to $\sigma_{i}^{\prime}$. This contradicts the hypothesis that the best response of player $j$ changes from $\sigma_{j}$ to $\sigma_{j}^{\prime}$ when player $i$ moves from $\sigma_{i}$ to $\sigma_{i}^{\prime}$.

Hence we restrict our attention to the case where $\sigma_{i}$ contains both $u$ and $w$. Let $\sigma_{i}^{\prime} \backslash \sigma_{i}$ and $\sigma_{i} \backslash \sigma_{i}^{\prime}$ be the segments where $\sigma_{i}$ and $\sigma_{i}^{\prime}$ deviate from each other. Since $\sigma_{i}^{\prime}$ does not contain either $u$ or $w$ (or both) and $\sigma_{i}$ contains both $u$ and $w, \sigma_{i}^{\prime} \backslash \sigma_{i}$ and $\sigma_{i} \backslash \sigma_{i}^{\prime}$ are non-empty. By Proposition 2, $\sigma_{i}^{\prime} \backslash \sigma_{i}$ and $\sigma_{i} \backslash \sigma_{i}^{\prime}$ are (non-empty) internally disjoint paths with common endpoints, denoted $u^{\prime}$ and $w^{\prime}$. Their first endpoint $u^{\prime}$ appears no later than $u$ and their last endpoint $w^{\prime}$ appears no sooner than $w$ on $\sigma_{i}$ and $\sigma_{i}^{\prime}$ (see e.g. Fig. 1.e). Furthermore, either $u$ is different from $u^{\prime}$ or $w$ is different from $w^{\prime}$ (or both). By Proposition 2 and since $\sigma_{i}$ deviates from at least one of $p$ and $p^{\prime}$ between $u$ and $w$, there is a unique path $\sigma_{i}\left[u^{\prime}, u\right]$ between $u$ and $u^{\prime}$ and a unique path $\sigma_{i}\left[w, w^{\prime}\right]$ between $w$ and $w^{\prime}$. Let $z=\sigma_{i}\left[u^{\prime}, u\right] \cup \sigma_{i}\left[w, w^{\prime}\right]$. We highlight that both $\sigma_{i}\left[u^{\prime}, u\right]$ and $\sigma_{i}\left[w, w^{\prime}\right]$ are included in strategies $\sigma_{j}$ and $\sigma_{j}^{\prime}$. In particular, $\sigma_{j}\left[u^{\prime}, w^{\prime}\right]=z \cup p$. Using the previous observations, we obtain that:

$$
\begin{aligned}
\sum_{e \in \sigma_{j}\left[u^{\prime}, w^{\prime}\right]} d_{e}\left(\sigma_{e}\right) & \geq \sum_{e \in z} d_{e}\left(\sigma_{e}\right)+\sum_{e \in p} d_{e}\left(\sigma_{e}^{\prime}\right) \\
& >\sum_{e \in z} d_{e}\left(\sigma_{e}\right)+\sum_{e \in p^{\prime}} d_{e}\left(\sigma_{e}^{\prime}+1\right) \\
& \geq \sum_{e \in z} d_{e}\left(\sigma_{e}\right)+\sum_{e \in p^{\prime} \cap \sigma_{i}} d_{e}\left(\sigma_{e}\right)+\sum_{e \in p^{\prime} \backslash \sigma_{i}} d_{e}\left(\sigma_{e}+1\right) \\
& \geq \sum_{e \in \sigma_{i}^{\prime}\left[u^{\prime}, w^{\prime}\right]} d_{e}\left(\sigma_{e}+1\right)
\end{aligned}
$$


The first inequality holds because the edges of $p$ are not included in $\sigma_{i}^{\prime}$, and the congestion of any edge not in $\sigma_{i}^{\prime}$ does not increase when player $i$ moves from $\sigma_{i}$ to $\sigma_{i}^{\prime}$ (i.e. $\forall e \notin \sigma_{i}^{\prime}, \sigma_{e} \geq \sigma_{e}^{\prime}$ ). The second inequality follows from (1). The third inequality holds because when player $i$ switches from $\sigma_{i}$ to $\sigma_{i}^{\prime}$ : (i) the congestion of any edge decreases by at most 1 (i.e. $\forall e, \sigma_{e}^{\prime} \geq \sigma_{e}-1$ ), and (ii) the congestion of any edge not in $\sigma_{i}$ does not decrease (i.e. $\forall e \notin \sigma_{i}, \sigma_{e}^{\prime} \geq \sigma_{e}$ ). For the fourth inequality, we observe that the left-hand side is equal to the individual cost of player $i$ on $\sigma_{i}\left[u^{\prime}, u\right] \cup p^{\prime} \cup \sigma_{i}\left[w, w^{\prime}\right]$ in $\sigma$ (i.e. when the configuration of the remaining players is $\sigma_{-i}$ ), and that the right-hand side is equal to the individual cost of player $i$ on $\sigma_{i}^{\prime}\left[u^{\prime}, w^{\prime}\right]$ in $\sigma$ (recall that $\sigma_{i}^{\prime}\left[u^{\prime}, w^{\prime}\right]$ and $\sigma_{i}\left[u^{\prime}, w^{\prime}\right]$ are edge disjoint). Since $\sigma_{i}^{\prime}$ is a best response of player $i$ to $\sigma_{-i}$, the former cost is not less than the latter.

Thus (4) implies that player $j$ can decrease her individual cost in $\sigma$ by switching from $\sigma_{j}\left[u^{\prime}, w^{\prime}\right]$ to $\sigma_{i}^{\prime}\left[u^{\prime}, w^{\prime}\right]$. This contradicts the hypothesis that $\sigma_{j}$ is a best response of player $j$ to $\sigma$. Since we have reached a contradiction in all different cases, this concludes the proof of the lemma.

By Lemma 1, once a player finds herself on her best response strategy, she will not have an incentive to deviate as long as the remaining players switch to their best improvement strategies. Hence we obtain the main result of this section:

Theorem 1. For any n-player symmetric congestion game on an extension-parallel network, every best improvement sequence reaches a pure Nash equilibrium in at most $n$ steps.

\section{Bounding the Inefficiency of Pure Nash Equilibria}

We proceed to establish an upper bound of $\rho(\mathcal{D})$ on the PoS of symmetric network congestion games and on the PoA of symmetric games on extension parallel networks. We start with a technical property of the configurations of symmetric network congestion games that minimize the potential function $\Phi$ (cf. Lemma 2). Based on this property and on the definition of $\beta(\mathcal{D})$, we prove that for symmetric network games, the total cost of any global minimum of $\Phi$ is at most $(1-\beta(\mathcal{D}))^{-1}=\rho(\mathcal{D})$ times the optimal total cost (cf. Lemma 3). Since a global minimum of $\Phi$ is also a local minimum, and thus a pure Nash equilibrium, we obtain that the PoS of symmetric network congestion games with latency functions in class $\mathcal{D}$ is at most $\rho(\mathcal{D})$ (cf. Theorem 2). Furthermore, we show that for symmetric games on extension-parallel networks, every pure Nash equilibria is a global minimum of the potential function $\Phi$ (cf. Lemma 4). Therefore, for symmetric congestion games on extension-parallel networks with latency functions in class $\mathcal{D}$, the PoA is at most $\rho(\mathcal{D})$ (cf. Theorem 3).

Lemma 2. Let $\Gamma$ be a symmetric network congestion game, and let $G(V, E)$ be the underlying $s-t$ network. An acyclic configuration $f$ of $\Gamma$ minimizes the potential function $\Phi$ if and only if for every configuration $g$ of $\Gamma$,

$$
\Delta(f, g) \equiv \sum_{e: f_{e}>g_{e}}\left(f_{e}-g_{e}\right) d_{e}\left(f_{e}\right)-\sum_{e: f_{e}<g_{e}}\left(g_{e}-f_{e}\right) d_{e}\left(f_{e}+1\right) \leq 0
$$


Proof. For the if-part, we observe that for all configurations $f, g$,

$$
\begin{aligned}
\Phi(f)-\Phi(g) & =\sum_{e: f_{e}>g_{e}} \sum_{i=g_{e}+1}^{f_{e}} d_{e}(i)-\sum_{e: f_{e}<g_{e}} \sum_{i=f_{e}+1}^{g_{e}} d_{e}(i) \\
& \leq \sum_{e: f_{e}>g_{e}}\left(f_{e}-g_{e}\right) d_{e}\left(f_{e}\right)-\sum_{e: f_{e}<g_{e}}\left(g_{e}-f_{e}\right) d_{e}\left(f_{e}+1\right)=\Delta(f, g)
\end{aligned}
$$

The inequality holds because for every edge $e$ with $f_{e}>g_{e}, \sum_{i=g_{e}+1}^{f_{e}} d_{e}(i)$ consists of $f_{e}-g_{e}$ terms no greater than $d_{e}\left(f_{e}\right)$, and for every edge $e$ with $f_{e}<g_{e}, \sum_{i=f_{e}+1}^{g_{e}} d_{e}(i)$ consists of $g_{e}-f_{e}$ terms no less than $d_{e}\left(f_{e}+1\right)$. Therefore, if for all configurations $g$ of $\Gamma, \Delta(f, g) \leq 0$, then $\Phi(f) \leq \Phi(g)$ for all configurations $g$, which implies that $f$ is a global minimum of the potential function $\Phi$.

For the converse, let $f$ be an acyclic configuration that minimizes $\Phi$, and let $g$ be any acyclic configuration of $\Gamma$. The assumption that $g$ is acyclic can be made without loss of generality, since any configuration $g^{\prime}$ can be turned into an acyclic configuration $g$ with $g_{e} \leq g_{e}^{\prime}$ for all $e \in E$, which implies that $\Delta\left(f, g^{\prime}\right) \leq \Delta(f, g)$. In the following, we assume that the configurations $f$ and $g$ are not identical, and consider the corresponding feasible $s-t$ flows.

Let $\hat{G}(V, \hat{E})$ be the network of the flow $g-f$. In particular, for each edge $(u, w) \in E, \hat{E}$ contains a forward edge $(u, w)$ with flow $g_{(u, w)}-f_{(u, w)}$ if $g_{(u, w)}>f_{(u, w)}$, a backward edge $(w, u)$ with flow $f_{(u, w)}-g_{(u, w)}$ if $g_{(u, w)}<f_{(u, w)}$, and no edge between $u$ and $w$ if $f_{(u, w)}=g_{(u, w)}$. For a (directed) cycle $C$ in $\hat{G}$, let

$$
C^{+} \equiv\left\{(u, w) \in E:(u, w) \in C \text { and } g_{(u, w)}>f_{(u, w)}\right\}
$$

be the set of edges of the original network $G$ corresponding to the forward edges of $C$, let

$$
C^{-} \equiv\left\{(u, w) \in E:(w, u) \in C \text { and } g_{(u, w)}<f_{(u, w)}\right\}
$$

be the set of edges of the original network $G$ (with their directions as in $E$ ) corresponding to the backward edges of $C$, and let

$$
\operatorname{cost}_{f}(C) \equiv \sum_{e \in C^{+}} d_{e}\left(f_{e}+1\right)-\sum_{e \in C^{-}} d_{e}\left(f_{e}\right)
$$

be the cost (with respect to $f$ ) of cycle $C$. The only-if-part of the lemma follows from the following claims: (i) if $f$ is acyclic and a global minimum of the potential function $\Phi$, then for every acyclic configuration $g$, the network $\hat{G}$ of the flow $g-f$ does not contain any cycles $C$ with $\operatorname{cost}_{f}(C)<0$, and (ii) if every cycle $C$ in the network $\hat{G}$ of the flow $g-f$ has $\operatorname{cost}_{f}(C) \geq 0$, then $\Delta(f, g) \leq 0$.

We first establish claim (ii), namely that if $\hat{G}$ does not contain any cycles of negative cost (with respect to $f$ ), then $\Delta(f, g) \leq 0$. Since $f$ and $g$ are acyclic $s-t$ flows of the same volume, a flow decomposition of $g-f$ yields only cycles and no paths of $\hat{G}$. Let $\left\{C_{1}, \ldots, C_{k}\right\}$ be the set of (directed, simple) cycles of $\hat{G}$ produced by the standard flow decomposition of $g-f$ (see e.g. the algorithm in [3, Theorem 3.5]), and let $s_{i}$ be the amount of flow carried by each cycle $C_{i}$ in the decomposition of $g-f$. Since $g$ and $f$ are acyclic $s-t$ flows, every cycle $C_{i}$ contains at least one forward and at least one backward edge (i.e. both $C_{i}^{+}$and $C_{i}^{-}$are non-empty).

By the properties of the standard flow decomposition, $\bigcup_{i \in[k]} C_{i}^{+}=\left\{e \in E: g_{e}>f_{e}\right\}$ (i.e. the union of $C_{i}^{+}$'s corresponds to the set of forward edges of $\hat{G}$ ), and $\bigcup_{i \in[k]} C_{i}^{-}=\left\{e \in E: g_{e}<f_{e}\right\}$ 
(i.e. the union of $C_{i}^{- \text {' }}$ s corresponds to the set of backward edges of $\hat{G}$ ). Moreover, for every forward edge $(u, w) \in \hat{E}, \sum_{i:(u, w) \in C_{i}^{+}} s_{i}=g_{(u, w)}-f_{(u, w)}$, and for every backward edge $(w, u) \in \hat{E}$, $\sum_{i:(u, w) \in C_{i}^{-}} s_{i}=f_{(u, w)}-g_{(u, w)}$. Therefore,

$$
\begin{aligned}
\Delta(f, g) & =\sum_{e: f_{e}>g_{e}}\left(f_{e}-g_{e}\right) d_{e}\left(f_{e}\right)-\sum_{e: f_{e}<g_{e}}\left(g_{e}-f_{e}\right) d_{e}\left(f_{e}+1\right) \\
& =\sum_{i=1}^{k} s_{i} \underbrace{\left(\sum_{e \in C_{i}^{-}} d_{e}\left(f_{e}\right)-\sum_{e \in C_{i}^{+}} d_{e}\left(f_{e}+1\right)\right)}_{=-\operatorname{cost}_{f}\left(C_{i}\right)} \\
& =-\sum_{i=1}^{k} s_{i} \operatorname{cost}_{f}\left(C_{i}\right)
\end{aligned}
$$

Hence, if $\hat{G}$ does not contain any (simple) cycles of negative cost (with respect to $f$ ), then for every cycle $C_{i}$ in the flow decomposition of $g-f, \operatorname{cost}_{f}\left(C_{i}\right) \geq 0$, which implies that $\Delta(f, g) \leq 0$. This concludes the proof of claim (ii).

We proceed to establish claim (i), namely that if $f$ is a global minimum of the potential function $\Phi$, then $\hat{G}$ does not contain any cycles of negative cost (with respect to $f$ ). For sake of contradiction, we assume that there is a (directed) cycle $C$ in $\hat{G}$ with $\operatorname{cost}_{f}(C)<0$. Given the flow $f$ and the cycle $C$, we construct a feasible $s-t$ flow $f^{\prime}$ by increasing the flow by one unit on the edges of $C^{+}$(i.e. $f_{e}^{\prime}=f_{e}+1$ on all $e \in C^{+}$), decreasing the flow by one unit on the edges of $C^{-}$(i.e. $f_{e}^{\prime}=f_{e}-1$ on all $e \in C^{-}$), and keeping the same flow on the remaining edges (i.e. $f_{e}^{\prime}=f_{e}$ on all $\left.e \in E \backslash\left(C^{+} \cup C^{-}\right)\right)$. By construction, the network of the flow $g-f^{\prime}$ can be obtained from $\hat{G}$ (i.e. the network of the flow $g-f$ ) by decreasing the flow by one unit on all edges of $C$. Since $f$ and $g$ are feasible acyclic $s-t$ flows, $f^{\prime}$ is a feasible acyclic $s-t$ flow (and thus corresponds to a configuration of $\Gamma$ ). By construction, the potential value of $f^{\prime}$ is $\Phi\left(f^{\prime}\right)=\Phi(f)+\operatorname{cost}_{f}(C)<\Phi(f)$, because $\operatorname{cost}_{f}(C)<0$, which contradicts the hypothesis that $f$ is a global minimum of the potential function $\Phi$. This concludes the proof of claim (i), and the proof of the lemma.

The following lemma bounds the total cost of configurations (of symmetric network games) that minimize the potential function $\Phi$ in terms of the optimal total cost. The proof follows from the definition of $\rho(\mathcal{D})$ and Lemma 2.

Lemma 3. Let $\Gamma$ be a symmetric network congestion game with latency functions in class $\mathcal{D}$, let $f$ be an acyclic configuration of $\Gamma$ that minimizes the potential function $\Phi$, and let o be the optimal configuration. Then, $C(f) \leq \rho(D) C(o)$.

Proof. Let $G(V, E)$ be the network underlying the definition of $\Gamma$. For every edge $e$ with $f_{e}>o_{e}$,

$$
\begin{aligned}
f_{e} d_{e}\left(f_{e}\right) & =o_{e} d_{e}\left(f_{e}\right)+\left(f_{e}-o_{e}\right) d_{e}\left(f_{e}\right) \\
& \leq o_{e} d_{e}\left(o_{e}\right)+\beta(\mathcal{D}) f_{e} d_{e}\left(f_{e}\right)+\left(f_{e}-o_{e}\right) d_{e}\left(f_{e}\right)
\end{aligned}
$$

For the inequality, we use that $o_{e}\left(d_{e}\left(f_{e}\right)-d_{e}\left(o_{e}\right)\right) \leq \beta(\mathcal{D}) f_{e} d_{e}\left(f_{e}\right)$, which follows from the definition of $\beta(\mathcal{D})$, since $f_{e}>o_{e}$ and $d_{e}(x) \in \mathcal{D}$. 
On the other hand, for every edge $e$ with $f_{e}<o_{e}$,

$$
\begin{aligned}
f_{e} d_{e}\left(f_{e}\right) & =o_{e} d_{e}\left(o_{e}\right)-o_{e} d_{e}\left(o_{e}\right)+f_{e} d_{e}\left(f_{e}\right) \\
& \leq o_{e} d_{e}\left(o_{e}\right)-\left(o_{e}-f_{e}\right) d_{e}\left(f_{e}+1\right)
\end{aligned}
$$

The inequality follows from $d_{e}\left(f_{e}\right) \leq d_{e}\left(f_{e}+1\right)$ and $d_{e}\left(f_{e}+1\right) \leq d_{e}\left(o_{e}\right)$, because the latency functions are non-decreasing and $f_{e}+1 \leq o_{e}$ (recall that $o_{e}$ and $f_{e}$ are integral).

Using (7), (8), and Lemma 2, we obtain that:

$$
\begin{aligned}
C(f) & \leq C(o)+\beta(\mathcal{D}) \sum_{e: f_{e}>o_{e}} f_{e} d_{e}\left(f_{e}\right)+\overbrace{\sum_{e: f_{e}>o_{e}}\left(f_{e}-o_{e}\right) d_{e}\left(f_{e}\right)-\sum_{e: f_{e}<o_{e}}\left(o_{e}-f_{e}\right) d_{e}\left(f_{e}+1\right)}^{=\Delta(f, o) \leq 0, \text { by Lemma } 2} \\
& \leq C(o)+\beta(\mathcal{D}) C(f),
\end{aligned}
$$

which implies that $C(f) \leq(1-\beta(\mathcal{D}))^{-1} C(o)=\rho(\mathcal{D}) C(o)$. For the first inequality, we apply (7) to every edge $e$ with $f_{e}>o_{e}$ and (8) to every edge $e$ with $f_{e}<o_{e}$. For the last inequality, since $f$ is an acyclic configuration that minimizes the potential function $\Phi$ and $o$ is a configuration of $\Gamma$, Lemma 2 implies that $\Delta(f, o) \leq 0$.

Every symmetric network congestion game admits an acyclic configuration that minimizes the potential function $\Phi$. Since the global minimum of $\Phi$ is also a local minimum, and thus a pure Nash equilibrium, Lemma 3 implies that every symmetric network game with latencies in class $\mathcal{D}$ admits a pure Nash equilibrium of total cost at most $\rho(\mathcal{D})$ times the optimal total cost. Hence we obtain the following upper bound on the (pure) Price of Stability of symmetric network congestion games.

Theorem 2. For any symmetric network congestion game with latency functions in class $\mathcal{D}$, the $\operatorname{PoS}$ is at most $\rho(\mathcal{D})$.

For symmetric games on extension-parallel networks, configurations are acyclic and every pure Nash equilibrium is a global minimum of the potential function $\Phi$ (cf. Lemma 4). Therefore, by Lemma 3, for symmetric games on extension-parallel networks with latency functions in class $\mathcal{D}$, the total cost of any pure Nash equilibrium is at most $\rho(\mathcal{D})$ times the optimal total cost. Hence we obtain the following upper bound on the pure Price of Anarchy of symmetric congestion games on extension-parallel networks.

Theorem 3. For symmetric congestion games on extension-parallel networks with latency functions in class $\mathcal{D}$, the PoA is at most $\rho(\mathcal{D})$.

To conclude the proof of Theorem 3, we have to show that:

Lemma 4. Every pure Nash equilibrium of a symmetric congestion game on an extension-parallel network is a global minimum of the potential function $\Phi$.

Proof $^{5}$. Let $\Gamma$ be a symmetric congestion game on an extension-parallel network $G(V, E)$, and let $f$ be any pure Nash equilibrium of $\Gamma$. We show that for every configuration $g$ of $\Gamma, \Delta(f, g) \leq 0$.

\footnotetext{
${ }^{5}$ Lemma 4 is implicit in the work of Holzman and Law-Yone (see [19, Theorem 6.1]). However, the proof of Theorem 6.1 is omitted from [19]. Hence, for sake of completeness and because our framework is different from that of [19], we give a complete proof of Lemma 4 using techniques different from those in [19].
} 
Then by Lemma 2 (if-part), $\Phi(f) \leq \Phi(g)$ for all configurations $g$, which implies that $f$ is a global minimum of the potential function $\Phi$. To establish that $\Delta(f, g) \leq 0$, we consider the network $\hat{G}(V, \hat{E})$ of the flow $g-f$, and show that if $f$ is a pure Nash equilibrium, the cost (with respect to $f$ ) of any (directed) simple cycle in $\hat{G}$ is non-negative (i.e. this corresponds to claim (i) in the proof of Lemma 2, only-if-part, claim (ii) holds with exactly the same proof).

In the following, we use the same notation as in the proof of Lemma 2. We recall that $\Delta(f, g)=$ $-\sum_{i=1}^{k} s_{i} \operatorname{cost}_{f}\left(C_{i}\right)$ (see (6)), where $\left\{C_{1}, \ldots, C_{k}\right\}$ is the set of (directed, simple) cycles produced by the standard flow decomposition of $g-f$, and $s_{i}$ is the amount of flow carried by each cycle $C_{i}$ in the decomposition of $f-g$ (recall that $f, g$ are acyclic, as configurations of a symmetric game on an extension-parallel network). Therefore to establish that $\Delta(f, g) \leq 0$, it suffices to prove that for every (simple) cycle $C_{i}$ in the decomposition of $g-f, \operatorname{cost}_{f}\left(C_{i}\right) \geq 0$.

In fact, we prove that for every simple cycle $C$ of $\hat{G}$, $\operatorname{cost}_{f}(C) \geq 0$, which by the discussion above, implies the lemma. The crux of the proof is to show that due to extension-parallel structure of the network, every simple cycle $C$ of $\hat{G}$ contains two vertices $u, w$ such that $C^{+}$and $C^{-}$are two internally disjoint $u-w$ paths in the original network $G$ (cf. Proposition 4). Then the claim follows from the hypothesis that $f$ is a pure Nash equilibrium. More precisely, since $C^{-}$consists of backward edges only, $f_{e}>0$ for all $e \in C^{-}$. Hence by Proposition 3, there is a player $i$ whose strategy in $f$ includes $C^{-}$. Therefore, $\sum_{e \in C^{-}} d_{e}\left(f_{e}\right) \leq \sum_{e \in C^{+}} d_{e}\left(f_{e}+1\right)$, since otherwise player $i$ could switch from $C^{-}$to $C^{+}$between $u$ and $w$ and improve her individual cost, which contradicts the hypothesis that $f$ is a pure Nash equilibrium. Consequently, for every simple cycle $C$ of $\hat{G}$, $\operatorname{cost}_{f}(C) \geq 0$.

To conclude the proof of the lemma, we have to show that the forward and the backward part of every cycle $C$ in $\hat{G}$ correspond to two internally paths in the extension-parallel network $G$.

Proposition 4. Let $G$ be any extension-parallel $s-t$ network, let $f, g$ be any acyclic $s-t$ flows, let $\hat{G}$ be the network of the flow $g-f$, and let $C$ be any simple cycle of $\hat{G}$. Then there exist vertices $u, w$ on $C$ such that $C^{+}$and $C^{-}$are two internally disjoint $u-w$ paths in $G$.

Proof. We first establish a property of $\hat{G}$ required in the proof of Proposition 4.

Proposition 5. Let $G$ be any extension-parallel $s-t$ network, let $f, g$ be any acyclic $s-t$ flows, and let $\hat{G}$ be the network of the flow $g-f$. Then any (simple) $s-t$ path in $\hat{G}$ consists of forward edges only, and any (simple) $t-s$ path in $\hat{G}$ consists of backward edges only.

Proof. We prove only the first part, namely that any (simple) $s-t$ path in $\hat{G}$ consists of forward edges only. The second part concerning $t-s$ paths follows by applying the first part to the flow $f-g$.

The proof is by induction on the extension-parallel structure of the network. The proposition holds if the network consists of a single edge $(s, t)$. We inductively assume that the proposition holds for any extension-parallel network with less edges than $G$.

If $G$ is the parallel composition of two extension-parallel $s-t$ networks $G_{1}$ and $G_{2}$, since $g$ (resp. $f$ ) is an acyclic $s-t$ flow, it can be decomposed into an acyclic $s-t$ flow $g^{1}$ (resp. $f^{1}$ ) going through $G_{1}$, and an acyclic $s-t$ flow $g^{2}$ (resp. $f^{2}$ ) going through $G_{2}$. More precisely, $g^{1}$ (resp. $f^{1}$ ) is defined on $G_{1}$ only and is equal to $g_{e}$ (resp. $f_{e}$ ) on every edge $e$ of $G_{1}$. Similarly, $g^{2}$ (resp. $f^{2}$ ) is defined on $G_{2}$ only and is equal to $g_{e}$ (resp. $f_{e}$ ) on every edge $e$ of $G_{2}$. Let $\hat{G}_{1}$ (resp. $\hat{G}_{2}$ ) be the network of the flow $g^{1}-f^{1}$ (resp. $g^{2}-f^{2}$ ). Then $\hat{G}$ is the parallel composition of $\hat{G}_{1}$ and $\hat{G}_{2}$, and any $s-t$ path in 
$\hat{G}$ goes through either $\hat{G}_{1}$ or $\hat{G}_{2}$. The proposition follows from the induction hypothesis stating that $s-t$ paths in $\hat{G}_{1}$ and $\hat{G}_{2}$ consist of forward edges only.

If $G$ is the series composition of an edge $\left(s, s_{1}\right)$ and an extension-parallel $s_{1}-t$ network $G_{1}$, we assume that $g_{\left(s, s_{1}\right)}>f_{\left(s, s_{1}\right)}$ (i.e. that $\left(s, s_{1}\right)$ is a forward edge in $\hat{G}$ ), since $\hat{G}$ does not contain any $s-t$ path otherwise. Let $g^{1}$ (resp. $f^{1}$ ) be the flow obtained from $g$ (resp. $f$ ) by ignoring the (flow on) edge $\left(s, s_{1}\right)$. Then $g^{1}$ (resp. $f^{1}$ ) is an acyclic $s_{1}-t$ flow on $G_{1}$ with $g_{e}^{1}=g_{e}$ (resp. $f_{e}^{1}=f_{e}$ ) on every edge $e$ of $G_{1}$. Let $\hat{G}_{1}$ be the network of the flow $g^{1}-f^{1} . \hat{G}$ is the series composition of the forward edge $\left(s, s_{1}\right)$ and $\hat{G}_{1}$. Thus any $s-t$ path in $\hat{G}$ begins with the forward edge $\left(s, s_{1}\right)$ and continues with a $s_{1}-t$ path in $\hat{G}_{1}$. The proposition follows from the induction hypothesis stating that $s_{1}-t$ paths in $\hat{G}_{1}$ consist of forward edges only. Exactly the same argument applies when $G$ is the series composition of an extension-parallel $s-t_{1}$ network $G_{1}$ an edge $\left(t_{1}, t\right)$.

We proceed to establish Proposition 4, namely that for every simple cycle $C$ of $\hat{G}$, there are vertices $u, w$ on $C$ such that $C^{+}$and $C^{-}$are two internally disjoint $u-w$ paths in $G$. The proof is by induction on the extension-parallel structure of the network. If the network consists of a single edge $(s, t)$, the proposition holds trivially because $\hat{G}$ does not contain any cycles. We inductively assume that the proposition holds for any extension-parallel network with less edges than $G$.

If $G$ is the series composition of an edge $\left(s, s_{1}\right)$ and an extension-parallel $s_{1}-t$ network $G_{1}$, no cycle of $\hat{G}$ contains $s$. Thus the vertex $s$ and the edge $\left(s, s_{1}\right)$ can be ignored. Let $g^{1}$ (resp. $\left.f^{1}\right)$ be the flow obtained from $g$ (resp. $f$ ) by ignoring the (flow on) edge $\left(s, s_{1}\right)$. Then $g^{1}$ (resp. $f^{1}$ ) is an acyclic $s_{1}-t$ flow on $G_{1}$ with $g_{e}^{1}=g_{e}$ (resp. $f_{e}^{1}=f_{e}$ ) on every edge $e$ of $G_{1}$. Let $\hat{G}_{1}$ be the network of the flow $g^{1}-f^{1}$. Then every simple cycle $C$ of $\hat{G}$ is also a simple cycle of $\hat{G}_{1}$ (and vice versa), and the proposition follows from the induction hypothesis. The same argument applies when $G$ is the series composition of an extension-parallel $s-t_{1}$ network $G_{1}$ and an edge $\left(t_{1}, t\right)$.

If $G$ is the parallel composition of two extension-parallel $s-t$ networks $G_{1}$ and $G_{2}$, since $g$ (resp. $f$ ) is an acyclic $s-t$ flow, it can be decomposed into an acyclic $s-t$ flow $g^{1}$ (resp. $f^{1}$ ) going through $G_{1}$, and an acyclic $s-t$ flow $g^{2}$ (resp. $f^{2}$ ) going through $G_{2}$. More precisely, $g^{1}$ (resp. $f^{1}$ ) is defined only on $G_{1}$ and is equal to $g_{e}$ (resp. $f_{e}$ ) on every edge $e$ of $G_{1}$. Similarly, $g^{2}$ (resp. $f^{2}$ ) is defined only on $G_{2}$ and is equal to $g_{e}$ (resp. $f_{e}$ ) on every edge $e$ of $G_{2}$. Let $\hat{G}_{1}$ (resp. $\hat{G}_{2}$ ) be the network of the flow $g^{1}-f^{1}$ (resp. $g^{2}-f^{2}$ ). Then $\hat{G}$ is the parallel composition of $\hat{G}_{1}$ and $\hat{G}_{2}$. Therefore every simple cycle $C$ of $\hat{G}$ either lies entirely inside $\hat{G}_{1}$ (or $\hat{G}_{2}$ ) or consists of an $s-t$ path $p^{+}$in $\hat{G}_{1}$ (resp. $\hat{G}_{2}$ ) followed by a $t-s$ path $p^{-}$in $\hat{G}_{2}$ (resp. $\hat{G}_{1}$ ). In the former case, the proposition follows from the induction hypothesis. In the latter case, by Proposition 5, the $s-t$ path $p^{+}$consists of forward edges only and the $t-s$ path $p^{-}$consists of backward edges only. Hence $C^{+}=p^{+}$and $C^{-}=\left\{(u, w) \in E:(w, u) \in p^{-}\right\}$, and $C^{+}$and $C^{-}$are two internally disjoint $s-t$ paths in $G$.

This concludes the proof of Lemma 4.

Remark. The PoA may be greater than $\rho(\mathcal{D})$ even for series-parallel networks with linear latencies. For example, let us consider the 3-player game in Fig. 2. Since the latency functions are linear, $\rho=$ $4 / 3$. In the optimal configuration $o$, every edge has congestion 1 and the total cost is $C(o)=11$ (e.g. an optimal configuration is obtained by assigning the first player to the upper path $\left(e_{1}, e_{3}, e_{5}\right)$, the second player to the middle path $\left(e_{2}, e_{4}, e_{6}\right)$, and the third player to the lower path $\left.\left(e_{7}\right)\right)$. On the other 


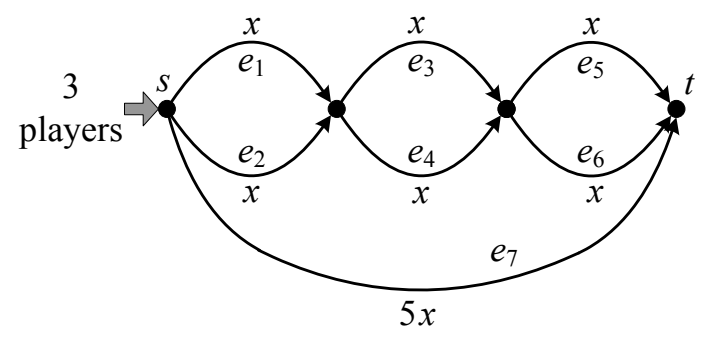

Fig. 2. A symmetric congestion game on a series-parallel network with linear latency functions and PoA greater than $4 / 3$.

hand, there is a pure Nash equilibrium $f$ where the first player is assigned to $\left(e_{1}, e_{3}, e_{6}\right)$, the second player to $\left(e_{1}, e_{4}, e_{5}\right)$, and the third player to $\left(e_{2}, e_{3}, e_{5}\right)$. Each player incurs an individual cost of 5 and does not have an incentive to deviate to $e_{7}$. The total cost is $C(f)=15$ and the $\operatorname{PoA}$ is $15 / 11>4 / 3$. In addition, we observe that $f$ is not a global minimum of the potential function $\Phi$ (this follows from Lemma 3 as well). In fact, $\Phi(f)=12$, while the optimal configuration has $\Phi(o)=11$ and is the global minimum of $\Phi$ (and thus a pure Nash equilibrium). Although Proposition 4 holds for the more general class of series-parallel networks, Lemma 4 fails in this example because Proposition 3 does not hold for series-parallel networks (and for the network in Fig. 2 in particular).

\section{Conclusions}

In this work, we considered symmetric congestion games on extension-parallel networks, and established two remarkable properties previously known only for parallel-link games. In particular, we proved that for arbitrary (non-negative and non-decreasing) latency functions, any best improvement sequence reaches a pure Nash equilibrium in at most $n$ steps, and that for latency functions in class $\mathcal{D}$, the PoA is at most $\rho(\mathcal{D})$. Moreover, our analysis implies that for symmetric network games with latency functions in class $\mathcal{D}$, the $\operatorname{PoS}$ is at most $\rho(\mathcal{D})$.

An interesting direction for further research is to investigate whether similar properties hold for more general classes of symmetric network congestion games. For instance, it would be quite interesting to establish a polynomial upper bound on the length of (certain) best improvement sequences for symmetric games on series-parallel networks. Lemma 1 may be a good starting point in this direction, since the analysis of several of the cases considered in the proof also applies to series-parallel networks. As for the inefficiency of Nash equilibria, it would be interesting to establish matching upper and lower bounds on the pure Price of Anarchy for symmetric congestion games on series-parallel networks and on general networks, especially if these bounds turn up to be considerably better than the known bounds for general congestion games (see e.g. $[6,9,4]$ ).

\section{References}

1. H. Ackermann, H. Röglin, and B. Vöcking. On the Impact of Combinatorial Structre on Congestion Games. Proc. of the 47th IEEE Symp. on Foundations of Computer Science (FOCS ’06), pp. 613-622, 2006.

2. H. Ackermann, H. Röglin, and B. Vöcking. Pure Nash Equilibria in Player-Specific and Weighted Congestion Games. Proc. of the 2nd Workshop on Internet and Network Economics (WINE '06), LNCS 4286, pp. 50-61, 2006.

3. R.K. Ahuja, T.L. Magnanti, and J.B. Orlin. Network Flows: Theory, Algorithms, and Applications. Prentice Hall, 1993. 
4. S. Aland, D. Dumrauf, M. Gairing, B. Monien, and F. Schoppmann. Exact Price of Anarchy for Polynomial Congestion Games. Proc. of the 23st Annual Symposium on Theoretical Aspects of Computer Science (STACS '06), LNCS 3884, pp. 218-229, 2006.

5. E. Anshelevich, A. Dasgupta, J. Kleinberg, É. Tardos, T. Wexler, and T. Roughgarden. The Price of Stability for Network Design with Fair Cost Allocation. Proc. of the 45th IEEE Symp. on Foundations of Computer Science (FOCS '04), pp. 295-304, 2004.

6. B. Awerbuch, Y. Azar, and A. Epstein. The Price of Routing Unsplittable Flow. Proc. of the 37th ACM Symp. on Theory of Computing (STOC '05), pp. 57-66, 2005.

7. I. Caragiannis, M. Flammini, C. Kaklamanis, P. Kanellopoulos, and L. Moscardelli. Tight Bounds for Selfish and Greedy Load Balancing. Submitted for journal publication, April 2007. Preliminary version in Proc. of the 33th International Colloquium on Automata, Languages and Programming (ICALP '06), LNCS 4051, pp. 311-322, 2006.

8. S. Chien and A. Sinclair. Convergece to Approximate Nash Equilibria in Congestion Games. Proc. of the 18th ACMSIAM Symposium on Discrete Algorithms (SODA '07), pp. 169-178, 2007.

9. G. Christodoulou and E. Koutsoupias. The Price of Anarchy of Finite Congestion Games. Proc. of the 37th ACM Symp. on Theory of Computing (STOC '05), pp. 67-73, 2005.

10. G. Christodoulou and E. Koutsoupias. On the Price of Anarchy and Stability of Correlated Equilibria of Linear Congestion Games. Proc. of the 13th European Symposium on Algorithms (ESA '05), LNCS 3669, pp. 59-70, 2005.

11. J.R. Correa, A.S. Schulz, and N.E. Stier Moses. Selfish Routing in Capacitated Networks. Mathematics of Operations Research, 29(4):961-976, 2004.

12. A. Epstein, M. Feldman, and Y. Mansour. Efficient Graph Topologies in Network Routing Games. Joint Workshop on Economics of Networked Systems and Incentive-Based Computing (NetEcon+IBC '07), 2007.

13. A. Epstein, M. Feldman, and Y. Mansour. Strong Equilibrium in Cost Sharing Connection Games. Proc. of the 8th ACM Conference on Electronic Commerce (EC '07), pp. 84-92, 2007.

14. E. Even-Dar, A. Kesselman, and Y. Mansour. Convergence Time to Nash Equilibria in Load Balancing. ACM Transactions on Algorithms, 3(3), 2007.

15. A. Fabrikant, C. Papadimitriou, and K. Talwar. The Complexity of Pure Nash Equilibria. Proc. of the 36th ACM Symp. on Theory of Computing (STOC '04), pp. 604-612, 2004.

16. D. Fotakis. Stackelberg Strategies for Atomic Congestion Games. Proc. of the 15th European Symposium on Algorithms (ESA '07), LNCS 4698, pp. 299-310, 2007.

17. M. Gairing, T. Lücking, M. Mavronicolas, B. Monien, and M. Rode. Nash Equilibria in Discrete Routing Games with Convex Latency Functions. Proc. of the 31th International Colloquium on Automata, Languages and Programming (ICALP '04), LNCS 3142, pp. 645-657, 2004.

18. M. Gairing, T. Lücking, B. Monien, and K. Tiemann. Nash Equilibria, the Price of Anarchy and the Fully Mixed Nash Equilibrium Conjecture. Proc. of the 32th International Colloquium on Automata, Languages and Programming (ICALP '05), LNCS 3580, pp. 51-65, 2005.

19. R. Holzman and N. Law-Yone. Strong Equilibrium in Congestion Games. Games and Economic Behaviour, 21:85-101, 1997.

20. R. Holzman and N. Law-Yone (Lev-tov). Network Structure and Strong Equilibrium in Route Selection Games. Mathematical Social Sciences, 46:193-205, 2003.

21. S. Ieong, R. McGrew, E. Nudelman, Y. Shoham, and Q. Sun. Fast and compact: A simple class of congestion games. 20th National Conference on Artificial Intellingence (AAAI '05), pp. 489-494, 2005.

22. E. Koutsoupias and C. Papadimitriou. Worst-case Equilibria. Proc. of the 16th Annual Symposium on Theoretical Aspects of Computer Science (STACS '99), LNCS 1563, pp. 404-413, 1999.

23. T. Lücking, M. Mavronicolas, B. Monien, and M. Rode. A New Model for Selfish Routing. Proc. of the 21st Annual Symposium on Theoretical Aspects of Computer Science (STACS '04), LNCS 2996, pp. 547-558, 2004.

24. I. Milchtaich. Network Topology and the Efficiency of Equilibrium. Games and Economic Behaviour, 57:321-346, 2006.

25. I. Milchtaich. The Equilbrium Existence Problem in Finite Network Congestion Games. Proc. of the 2nd Workshop on Internet and Network Economics (WINE '06), LNCS 4286, pp. 87-98, 2006.

26. R.W. Rosenthal. A Class of Games Possessing Pure-Strategy Nash Equilibria. International Journal of Game Theory, 2:65-67, 1973.

27. T. Roughgarden. The Price of Anarchy is Independent of the Network Topology. Journal of Computer and System Sciences, 67(2):341-364, 2003.

28. T. Roughdarden and É. Tardos. How Bad is Selfish Routing? Journal of the ACM, 49(2):236-259, 2002. 


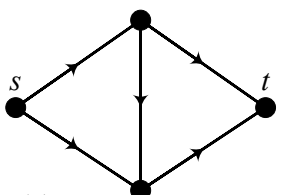

(a)

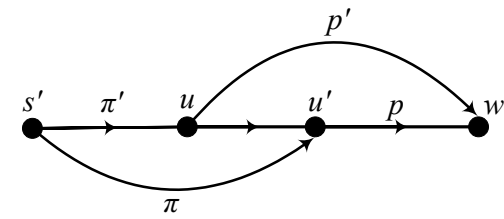

(b)

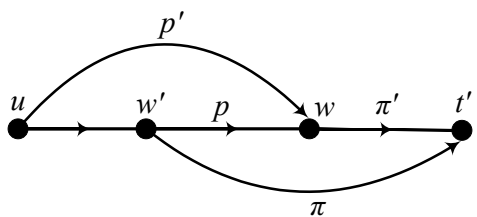

(c)

Fig. 3. (a) The $\theta$-network whose absence characterizes series-parallel $s-t$ networks. (b) and (c) If a path $\pi$ has some edges in common with $p$ (here $p$ and $\pi$ share the segment $\left(u^{\prime}, w\right)$ in (b) and the segment $\left(u, w^{\prime}\right)$ in (c)) and avoids either $u$ or $w$, it forms (together with $p, p^{\prime}$, and their extension to the source in (b) and to the sink in (c)) a network that includes the $\theta$-network as a topological minor, and thus it is not series-parallel.

\section{A Appendix}

\section{A.1 The Proof of Proposition 1}

We first recall that a $s-t$ network is series-parallel if and only if it does not contain a $\theta$-network with terminals of degree 2 (see Fig. 3.a) as a topological minor (see e.g. [24, Proposition 1].)

We observe that the proposition is trivial if $u=s$ and $w=t$. Hence, we assume that $u \neq s$. The case where $u=s$ and $w \neq t$ follows by symmetry.

Let $\pi$ be a $s-t$ path sharing some edges with $p$. We first argue by contradiction that $\pi$ does not intersect $p^{\prime}$ at any vertex other than $u$ and $w$. Let us assume that $\pi$ intersects $p^{\prime}$ at some vertex other than $u$ and $w$. Since $\pi$ has some edges in common with $p$, there is a segment of $\pi$ connecting a vertex of $p-\{u, w\}$ to a vertex of $p^{\prime}-\{u, w\}$ (or connecting a vertex of $p^{\prime}-\{u, w\}$ to a vertex of $p-\{u, w\}$ depending on whether $\pi$ first goes through an edge of $p$ and then intersects $p^{\prime}-\{u, w\}$ or the other way around). The endpoints of this segment of $\pi$ belong to $\left(p \cup p^{\prime}\right)-\{u, w\}$. Thus the endpoints of this segment of $\pi$ together with $p$ and $p^{\prime}$ form a $\theta$-network with terminals $u$ and $w$, a contradiction. Consequently $\pi$ does not intersect $p^{\prime}$ except possibly at $u$ and $w$.

We then prove that $\pi$ contains both $u$ and $w$. To reach a contradiction, we assume that $\pi$ does not contain $u$. Let $\pi^{\prime}$ be a path connecting $s$ to $u$, and let $s^{\prime}, s^{\prime} \neq u$, be the last common vertex of $\pi$ and $\pi^{\prime}$ before $u$ ( $s^{\prime}$ exists because $\pi$ and $\pi^{\prime}$ have a common source and $\pi$ does not contain $u$ ). Let also $u^{\prime}$, $u^{\prime} \neq u$, be the first common vertex of $\pi$ and $p$ after $u$ ( $u^{\prime}$ exists because $\pi$ intersects $p$ and $\pi$ does not contain $u$ ). Then, the vertices $s^{\prime}, u, u^{\prime}$, and $w$ along with $\pi^{\prime}$ (the segment from $s^{\prime}$ to $u$ ), $p$ (the segment from $u$ to $u^{\prime}$ and the segment from $u^{\prime}$ to $w$ ), $p^{\prime}$ (the segment from $u$ to $w$ ), and $\pi$ (the segment from $s^{\prime}$ to $u^{\prime}$ ) form the $\theta$-network of Fig. 3.b, which is a topological minor of $G$. This contradicts the hypothesis that $G$ is series-parallel network. If $\pi$ contains $u$ but not $w, w$ is different from $t$. Then the proposition follows from the previous argument by symmetry (see also Fig. 3.c).

\section{A.2 Proof of Lemma 1: Case I.b, $\sigma_{i}^{\prime} \cap p \neq \emptyset$ and $\left(\sigma_{i}^{\prime}[u, w] \backslash p\right) \cap \sigma_{i} \neq \emptyset$}

We first show that the congestion of the edges of $p^{\prime}$ does not change when player $i$ moves from $\sigma_{i}$ to $\sigma_{i}^{\prime}$. We recall that by Proposition $1, \sigma_{i}^{\prime}$ does not have any edges in common with $p^{\prime}$ and does not intersect $p^{\prime}$ at any vertex other than $u$ and $w$. In addition, since $u$ and $w$ are connected by two internally disjoint paths $p^{\prime}$ and $\sigma_{i}^{\prime}[u, w]$, and since $\sigma_{i}$ and $\sigma_{i}^{\prime}[u, w]$ have some edges in common, by Proposition $1, \sigma_{i}$ also 
contains $u$ and $w$ and does not have any edges in common with $p^{\prime}$. Hence the congestion of the edges of $p^{\prime}$ is the same in both $\sigma$ and $\sigma^{\prime}$.

Next we prove that the congestion of the edges of $p$ does not change when player $i$ moves from $\sigma_{i}$ to $\sigma_{i}^{\prime}$. Let $\pi=\sigma_{i}^{\prime}[u, w] \backslash p$ and $\pi^{\prime}=p \backslash \sigma_{i}^{\prime}[u, w]$ be the segments where $\sigma_{i}^{\prime}[u, w]$ and $p$ deviate from each other. Since $\pi \cap \sigma_{i} \neq \emptyset, \pi$ and $\pi^{\prime}$ are non-empty. Thus $\pi$ and $\pi^{\prime}$ are internally disjoint paths with common endpoints, denoted $u^{\prime}$ and $w^{\prime}$. Their first endpoint $u^{\prime}$ appears no sooner than $u$ and their last endpoint $w^{\prime}$ appears no later than $w$ on $\sigma_{i}^{\prime}$ and $p$. Since $\sigma_{i}$ has some edges in common with $\pi$, by Proposition 1, $\sigma_{i}$ contains $u^{\prime}$ and $w^{\prime}$ and does not intersect $\pi^{\prime}$ at any vertex other than $u^{\prime}$ and $w^{\prime}$. Furthermore, since all three paths $p, \sigma_{i}^{\prime}[u, w]$ and $\sigma_{i}[u, w]$ contain $u^{\prime}$ and $w^{\prime}$, and since $\sigma_{i}^{\prime}[u, w]$ and $\sigma_{i}[u, w]$ deviate from $p$ between $u^{\prime}$ and $w^{\prime}$, by Proposition 2, the three paths share their $u-u^{\prime}$ and $w^{\prime}-w$ segments (see also Fig. 1.d). Therefore, if $\sigma_{i}^{\prime}[u, w]$ and $\sigma_{i}[u, w]$ deviate from each other, this happens between $u^{\prime}$ and $w^{\prime}$. Consequently, the corresponding path segments $\sigma_{i}^{\prime}\left[u^{\prime}, w^{\prime}\right]$ and $\sigma_{i}\left[u^{\prime}, w^{\prime}\right]$ do not contain any edges of $p$. Thus the congestion of the edges of $p$ does not change when player $i$ switches from $\sigma_{i}$ to $\sigma_{i}^{\prime}$. 